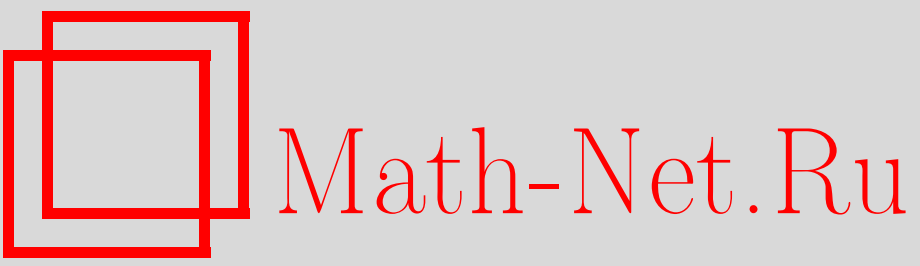

В. В. Тензина, Некоторые свойства топологического радикала Бэра колец с топологической размерностью Крулля, УМH, 2005, том 60, выпуск 2, 175-176

DOI: https://doi.org/10.4213/rm1422

Использование Общероссийского математического портала Math-Net.Ru подразумевает, что вы прочитали и согласны с пользовательским соглашением

http://www . mathnet.ru/rus/agreement

Параметры загрузки:

IP: 54.172 .240 .79

26 апреля 2023 г., 15:33:39 


\title{
НЕКОТОРЫЕ СВОЙСТВА ТОПОЛОГИЧЕСКОГО РАДИКАЛА БЭРА КОЛЕЦ С ТОПОЛОГИЧЕСКОЙ РАЗМЕРНОСТЬЮ КРУЛЛЯ
}

\author{
В. В. ТЕнЗИнА
}

В данной статье доказывается, что для некоторых типов колец топологический радикал Бэра не содержит односторонних топологически идемпотентных идеалов, хотя в общем случае топологический радикал Бэра даже может содержать единицу (см. [1]).

ОпРЕДЕЛЕНИЕ. Назовем топологический модуль топологически неприводимым, если он не содержит замкнутых собственных подмодулей.

ЛЕмма. Пусть $M$ - топологически неприводимыц $R$-модуль, и пусть $I-$ топологически $\Sigma$-нильпотентный идеал кольца $R$, тогда $I M=0$.

ДокАЗАтЕльство. Предположим, что $I M \neq 0$. Следовательно, существует такой элемент $m$ из $M$, что $I m \neq 0$. Но тогда $[\mathrm{Im}]=M$. Пусть для натурального числа $n$ выполняется $\left[I^{n-1} m\right]=M$. Докажем, что тогда $\left[I^{n} m\right]=M$. Имеем: $\left[I^{n} m\right] \supseteq\left[I \cdot I^{n-1} m\right] \supseteq[I] \cdot\left[I^{n-1} m\right] \supseteq$ $I \cdot M$. Отсюда $\left[I^{n} m\right] \supseteq[I M]=M$. Итак, для любого натуралшного $n$ справедливо $\left[I^{n} m\right]=M$. Фиксируем произвольную окрестность нуля $W$ в $M$. Тогда найдется такая окрестность нуля $W_{1}$, что $W_{1}+W_{1} \subseteq W$. В колье $R$ выберем окрестность $V$ такую, что $V \cdot m \subseteq W_{1}$. Так как $I-$ топологически $\Sigma$-нильпотентный идеал, то $\exists n \in \mathbb{N}: I^{n} \subseteq V$. Итак, $M=\left[I^{n} m\right] \subseteq[V \cdot m] \subseteq$ $\left[W_{1}\right] \subseteq W_{1}+W_{1} \subseteq W$, т.е. $M \subseteq \bigcap_{W \in \tau(M)} W=0$, но это противоречит $I M \neq 0$.

ПредЛоженИЕ. Топологический радикал Бэра топологического кольца $R$ обнуляет все топологически неприводимые $R$-модули.

ДоКАЗАТЕЛЬСТво. Пусть $M$ - топологически неприводимьй $R$-модуль. Введем следующее обозначение: $A(M)=\{r \in R: r M=0\}$. Из доказанной выше леммы следует, что фактор-кольцо $R / A(M)$ не содержит топологически $\Sigma$-нилшпотентных идеалов, так как модуль $M$ точен и неприводим над $R / A(M)$. Поэтому $\mathscr{L}(R) \subseteq A(M)$, т.е. $\mathscr{L}(R) M=0$.

Определим для топологического модуля $M$ топологическую размерность Крулля, которую будем обозначать через $\operatorname{top} K \operatorname{dim} M$, как девиацию частично упорядоченного множества всех замкнутых подмодулей с упорядочением по включению (см. [2]).

Теорема 1. Пусть топологическое кольцо $R$ обладает топологическим левым модулем $M$ с топологической размерностью Крулля. Тогда если левый идеал $J$ - топологически идемпотентен, т.е. $\left[J^{2}\right]_{J}=J$, и содержится в топологическом радикале Бәра, то $J M=0$.

ДокАЗАТЕЛЬСтво. Предварительно введем несколько понятий, связанных с топологической размерностью Крулля. Назовем топологический модуль $M$ с топологической размерностью Крулля топологически критическим, если для любого замкнутого подмодуля $N$ модуля $M$ вьполняется top $K \operatorname{dim} M / N<\operatorname{top} K \operatorname{dim} M$. Аналогично дискретному случаю (см. [3]) можно доказать, практически полностью повторяя доказательство, что каждый топологический модуль с топологической размерностью Крулля содержит какой-то топологически критический подмодуль. Для каждого порядкового $\gamma$ определим замкнутое множество $S_{\gamma}(M)$. Положим $S_{0}(M)=0$. Пусть уже определены все $S_{\alpha}(M)$ для каждого порядкового $\alpha<\beta$. Тогда $S_{\beta}(M)$ определим следующим образом: если $\beta$ - предельное, то $S_{\beta}(M)=\left[\bigcup_{\alpha<\beta} S_{\alpha}(M)\right]$, иначе существует порядковое $\beta-1$, в этом случае $S_{\beta}(M)$ представляет собой замыкание суммы всех замкнутых подмодулей $N$ таких, что фактор-модуль $N / S_{\beta-1}(M)$ является топологически критическим. Заметим, что $\alpha_{1}<\alpha_{2} \Leftrightarrow S_{\alpha_{1}}(M) \leqslant S_{\alpha_{2}}(M)$. Существует такое порядковое $\delta$, что для любого $\gamma>\delta$ справедливо $S_{\gamma}(M)=S_{\delta}(M)$. Так как любой топологический модуль с топологической размерностью Крулля содержит хотя бы один топологически критический подмодуль, то $M=S_{\delta}(M)$.

Пусть утверждение теоремы верно для всех топологических модулей с топологической размерностью Крулля меньше $\beta$. Докажем справедливость утверждения, если top $K \operatorname{dim} M=\beta$. 
Пусть $C$ - топологически $\beta$-критический подмодуль $M$. Для любого замкнутого подмодуля $C^{\prime}$ по предположению $J C \subseteq C^{\prime}$. Следовательно, $[J C]$ - минимальный замкнутый подмодуль в $C$ и поэтому является топологически неприводимьм модулем. По предыдущей лемме $J[J C]=0$, так как $J \subseteq \mathscr{L}(R)$. Следовательно, $[J C]=\left[J^{2} C\right]=[J[J C]]=0$. Таким образом, $J C=0$. Поэтому $J S_{1}(M)=0$. При помощи трансффинитной индукции можно доказать, что $J M=0$.

ТЕОРема 2. Топологический радикал Бәра кольца, имеющего топологическую размерность Крулля, не содержит ненулевых односторонних топологически идемпотентных идеалов.

СледСтвиЕ. Топологический радикал Бәра топологического кольца с топологической размерностью Крулля не содержит единицу.

\section{СПИСОК ЛИТЕРАТУРЫ}

[1] В. И. Арнаутов // Докл. АН СССР. 1964. Т. 157. № 1. С. 12-15. [2] В. В. Тензина // Фундам. прикл. матем. 2004. T. 10. № 3. C. 215-230. [3] R. Gordon, J. C. Robson // Mem. Amer. Math. Soc. 1973. № 133. P. 1-78.

Московский государственный университет им. М. В. Ломоносова
Представлено А. В. Михалевьм Принято редколлегией 25.01.2005 